\title{
A NOTE ON RESOLVENT CONVERGENCE ON A THIN DOMAIN
}

\author{
RICARDO P. SILVA
}

(Received 29 November 2012; accepted 20 April 2013; first published online 27 June 2013)

\begin{abstract}
In this paper we provide a new proof of strong convergence of resolvent operators associated with boundary value problems on thin domains.

2010 Mathematics subject classification: primary 35B25.

Keywords and phrases: thin domains, resolvent convergence.
\end{abstract}

\section{Introduction}

Since the pioneer work by Hale and Raugel [15], the investigation of the asymptotic behaviour of the nonlinear dynamics of semilinear reaction-diffusion equations in thin domains has received the attention of several authors, mainly interested in the continuity of global attractors with respect to specific metrics; see [1, 7, 10-19] and their references.

Arrieta and Carvalho [3] and Carvalho and Piskarev [9] systematised a process to obtain the continuity of global attractors with respect to singular perturbations. Their ideas are based on a rigorous study of convergence properties of the linear part of the equation. Motivated by their ideas, in this paper we present a simple and direct proof of the convergence of resolvent operators of the singular problem considered in [17]. Although we are aware that the result itself has been proved by other authors, for example [1, 17], the point is that our combination of ideas presented in [1, 4] opens up the possibility of treating other classes of singular problems, such as those in $[2,4,8]$.

The paper is organised as follows: in Section 2 we present the functional analytic setting of the problem and we state the main result, Theorem 2.1, which is proved in Section 3.

\section{Preliminaries}

Let $\Omega$ be a smooth and bounded domain in $\mathbb{R}^{m+n}$. A generic point of $\Omega$ will be denoted by $(x, y) \in \mathbb{R}^{m} \times \mathbb{R}^{n}$ and $\epsilon$ will represent a small positive parameter.

Partially supported by FAPESP \#2012/06753-8 and FUNDUNESP \#0135812, Brazil

(C) 2013 Australian Mathematical Publishing Association Inc. 0004-9727/2013 \$16.00 
Considering the squeezing operator $\Phi_{\epsilon}: \mathbb{R}^{m+n} \rightarrow \mathbb{R}^{m+n}$ defined by $\Phi_{\epsilon}(x, y)=(x, \epsilon y)$, we set up the thin domain $\Omega_{\epsilon}:=\Phi_{\epsilon}(\Omega), \epsilon \in(0,1)$, and we analyse convergence properties of the solutions of the family of linear equations

$$
\begin{gathered}
-\Delta u^{\epsilon}+u^{\epsilon}=f^{\epsilon} \quad \text { in } \Omega_{\epsilon}, \\
\frac{\partial u^{\epsilon}}{\partial \eta_{\epsilon}}=0 \quad \text { on } \partial \Omega_{\epsilon},
\end{gathered}
$$

where $\eta_{\epsilon}$ denotes the outward unitary normal vector field to $\partial \Omega_{\epsilon}$.

In order to capture the limiting behaviour of $u^{\epsilon}$ as $\epsilon \rightarrow 0$, we perform a dilatation of the domain $\Omega_{\epsilon}$ by a factor $\epsilon$ in the $y$-direction. Thus we obtain in $\Omega$ the equivalent equations

$$
\begin{array}{cc}
-\Delta_{x} u-\frac{1}{\epsilon^{2}} \Delta_{y} u+u=\hat{f}^{\epsilon} & \text { in } \Omega, \\
\nabla_{x} u \cdot \eta_{x}+\frac{1}{\epsilon^{2}} \nabla_{y} u \cdot \eta_{y}=0 & \text { on } \partial \Omega,
\end{array}
$$

where $\hat{f}^{\epsilon}(x, y)=f^{\epsilon}(x, \epsilon y)$, and $\eta=\left(\eta_{x}, \eta_{y}\right)$ is the outward unitary normal vector field to $\partial \Omega$.

The relation between the spaces of functions defined in $\Omega_{\epsilon}$ and those defined in $\Omega$ is given by

$$
\Phi_{\epsilon}^{*}: L^{2}\left(\Omega_{\epsilon}\right) \rightarrow L^{2}(\Omega), \quad u \mapsto u \circ \Phi_{\epsilon},
$$

an isomorphism which restricts an isomorphism from $H^{1}\left(\Omega_{\epsilon}\right)$ onto $H^{1}(\Omega)$.

Stressing the fact that the domains $\Omega_{\epsilon}$ vary in accordance with the parameter $\epsilon$, collapsing themselves to a lower dimensional subset as $\epsilon \rightarrow 0$, to preserve the relative capacity of a measurable subset of $\Omega_{\epsilon}$ we perform a dilatation of the $(m+n)$ dimensional Lebesgue measure by a factor $1 / \epsilon$. With this measure, namely

$$
\rho_{\epsilon}:=\frac{1}{\epsilon} \times \text { Lebesgue measure, }
$$

we introduce the Lebesgue and Sobolev spaces $L^{2}\left(\Omega_{\epsilon} ; \rho_{\epsilon}\right)$ and $H^{1}\left(\Omega_{\epsilon} ; \rho_{\epsilon}\right)$, respectively. By considering in $H^{1}(\Omega)$ the equivalent norm

$$
\|u\|_{\epsilon}:=\left(\int_{\Omega}\left(\left|\nabla_{x} u\right|^{2}+\frac{1}{\epsilon^{2}}\left|\nabla_{y} u\right|^{2}+|u|^{2}\right) d x d y\right)^{1 / 2},
$$

it follows that the isomorphism

$$
\Phi_{\epsilon}^{*}: H^{1}\left(\Omega_{\epsilon} ; \rho_{\epsilon}\right) \rightarrow H_{\epsilon}^{1}(\Omega)
$$

is indeed an isometry, where $H_{\epsilon}^{1}(\Omega):=\left(H^{1}(\Omega),\|\cdot\|_{\epsilon}\right)$. A similar observation can be made regarding $\Phi_{\epsilon}^{*}: L^{2}\left(\Omega_{\epsilon} ; \rho_{\epsilon}\right) \rightarrow L^{2}(\Omega)$.

We notice that $H_{\epsilon}^{1}(\Omega)$ is a Hilbert space with respect to the inner product

$$
\begin{gathered}
a_{\epsilon}: H_{\epsilon}^{1}(\Omega) \times H_{\epsilon}^{1}(\Omega) \rightarrow \mathbb{R}, \\
a_{\epsilon}(u, v):=\int_{\Omega}\left(\nabla_{x} u \cdot \nabla_{x} v+\frac{1}{\epsilon^{2}} \nabla_{y} u \cdot \nabla_{y} v+u v\right) d x d y .
\end{gathered}
$$


This inner product induces a self-adjoint unbounded linear operator with compact resolvent

$$
A_{\epsilon}: D\left(A_{\epsilon}\right) \subset L^{2}(\Omega) \rightarrow L^{2}(\Omega)
$$

defined by

$$
a_{\epsilon}(u, v)=\left\langle A_{\epsilon} u, v\right\rangle, \quad \forall v \in H_{\epsilon}^{1}(\Omega), \forall u \in D\left(A_{\epsilon}\right),
$$

where $\langle u, v\rangle=\int_{\Omega} u v d x d y$. It follows from the smoothness of $\partial \Omega$ that

$$
D\left(A_{\epsilon}\right)=\left\{u \in H^{2}(\Omega): \nabla_{x} u \cdot \eta_{x}+\frac{1}{\epsilon^{2}} \nabla_{y} u \cdot \eta_{y}=0 \text { almost everywhere in } \partial \Omega\right\},
$$

and

$$
A_{\epsilon} u=-\Delta_{x} u-\frac{1}{\epsilon^{2}} \Delta_{y} u+u, \quad \forall u \in D\left(A_{\epsilon}\right) .
$$

Now, for $u \in H^{1}(\Omega)$, we notice that

$$
a_{\epsilon}(u, u) \stackrel{\epsilon \rightarrow 0}{\longrightarrow} \begin{cases}\int_{\Omega}\left(\left|\nabla_{x} u\right|^{2}+|u|^{2}\right) d x d y & \text { if } \nabla_{y} u=0 \\ \infty & \text { if } \nabla_{y} u \neq 0 .\end{cases}
$$

Therefore, in order to establish a formal limit for $a_{\epsilon}$, it is necessary to work in a proper subspace of $H^{1}(\Omega) \times H^{1}(\Omega)$. In [17] the authors introduce the subspace $H_{s}^{1}(\Omega):=\left\{u \in H^{1}(\Omega): \nabla_{y} u=0\right.$ almost everywhere in $\left.\Omega\right\}$ as well as the bilinear form $a_{0}: H_{s}^{1}(\Omega) \times H_{s}^{1}(\Omega) \rightarrow \mathbb{R}$ given by

$$
a_{0}(u, v):=\int_{\Omega}\left(\nabla_{x} u \cdot \nabla_{x} v+u v\right) d x d y, \quad \forall u, v \in H_{s}^{1}(\Omega) .
$$

The subspace $H_{s}^{1}(\Omega)$ is an infinite dimensional closed subspace of $H^{1}(\Omega)$.

Defining $L_{s}^{2}(\Omega):={\overline{H_{s}^{1}(\Omega)}}^{L^{2}(\Omega)}$, it follows that $a_{0}$ defines a self-adjoint unbounded linear operator with compact resolvent

$$
A_{0}: D\left(A_{0}\right) \subset L_{s}^{2}(\Omega) \rightarrow L_{s}^{2}(\Omega)
$$

defined by

$$
a_{0}(u, v)=\left\langle A_{0} u, v\right\rangle, \quad \forall v \in H_{s}^{1}(\Omega), \forall u \in D\left(A_{0}\right) .
$$

Analogously, it follows from the smoothness of $\partial \Omega$ that

$$
D\left(A_{0}\right)=\left\{u \in H^{2}(\Omega) \cap H_{s}^{1}(\Omega): \frac{\partial u}{\partial \eta}=0 \text { almost everywhere in } \partial \Omega\right\},
$$

and

$$
A_{0} u=-\Delta_{x} u+u, \quad \forall u \in D\left(A_{0}\right)
$$


Now we have the elements to state the main result of this paper.

THeOREM 2.1. Given a bounded family $f^{\epsilon} \in L^{2}(\Omega)$, there exists $f^{0} \in L^{2}(\Omega)$ such that, up to subsequence,

$$
\lim _{\epsilon \rightarrow 0}\left\|A_{\epsilon}^{-1} f^{\epsilon}-A_{0}^{-1} f^{0}\right\|_{\epsilon}=0 .
$$

Remark 2.2. After these considerations, at the limit where $\epsilon=0$, we must seek solutions $u \in H_{s}^{1}(\Omega)$ of the equations

$$
\begin{gathered}
-\Delta_{x} u+u=f^{0} \quad \text { in } \Omega, \\
\frac{\partial u}{\partial \eta}=0 \quad \text { on } \partial \Omega .
\end{gathered}
$$

If each $x$-section $\Omega_{x}:=\left\{y \in \mathbb{R}^{n}:(x, y) \in \Omega\right\}$ is a connected set, then such solutions are of the form $u(x, y)=v(x)$ almost everywhere in $\Omega$, for a suitable function $v$ defined in $P(\Omega):=\left\{x \in \mathbb{R}^{m}: \Omega_{x} \neq \emptyset\right\}$. This justifies the expression 'reduction of dimension' in such problems. Details can be found in [17].

\section{Convergence results}

In this section we are concerned with convergence properties of solutions of (2.1). This means that, given $f^{\epsilon} \in L^{2}(\Omega)$, we will deal with the family of linear problems

$$
A_{\epsilon} u^{\epsilon}=f^{\epsilon}
$$

investigating convergence of $u^{\epsilon}$ as $\epsilon \rightarrow 0$.

At first glance, we are able to prove the following weak convergence result.

Lemma 3.1. Let $f^{\epsilon} \in L^{2}(\Omega)$ be such that $\left\|f^{\epsilon}\right\|_{L^{2}(\Omega)} \leq c$ for some constant $c$ not dependent on $\epsilon \in(0,1)$. There exists $f^{0} \in L_{s}^{2}(\Omega)$ and $u^{0}=A_{0}^{-1} f^{0} \in H_{s}^{1}(\Omega)$ such that, up to subsequence,

$$
A_{\epsilon}^{-1} f^{\epsilon} \stackrel{\epsilon \rightarrow 0}{\longrightarrow} u^{0}, \quad w-H^{1}(\Omega) \quad \text { and } \quad s-L^{2}(\Omega) .
$$

Proof. Setting $u^{\epsilon}=A_{\epsilon}^{-1} f^{\epsilon} \in H_{\epsilon}^{1}(\Omega)$,

$$
a_{\epsilon}\left(u^{\epsilon}, v\right)=\left\langle f^{\epsilon}, v\right\rangle, \quad \forall v \in H_{\epsilon}^{1}(\Omega) .
$$

In particular, taking $v=u^{\epsilon}$ as a test function, it follows from Hölder's inequality that

$$
\left\|u^{\epsilon}\right\|_{H^{1}(\Omega)}^{2} \leq a_{\epsilon}\left(u^{\epsilon}, u^{\epsilon}\right) \leq\left\|f^{\epsilon}\right\|_{L^{2}(\Omega)}\left\|u^{\epsilon}\right\|_{H^{1}(\Omega)},
$$

which means

$$
\left\|u^{\epsilon}\right\|_{H^{1}(\Omega)} \leq c, \quad \forall \epsilon \in(0,1) .
$$

Since $H^{1}(\Omega)$ is a reflexive space and $L^{2}(\Omega) \hookrightarrow H^{1}(\Omega)$ compactly, taking a subsequence if necessary, there exists $u^{0} \in H^{1}(\Omega)$ such that $u^{\epsilon} \stackrel{\epsilon \rightarrow 0}{\longrightarrow} u^{0}$, weakly 
in $H^{1}(\Omega)$ and strongly in $L^{2}(\Omega)$. Moreover, noticing that $a_{\epsilon}\left(u^{\epsilon}, u^{\epsilon}\right) \leq c^{2}$, one has that $\left\|\nabla_{y} u^{\epsilon}\right\|_{L^{2}(\Omega)^{n}} \leq c \epsilon$. Therefore, $\nabla_{y} u^{\epsilon} \stackrel{\epsilon \rightarrow 0}{\longrightarrow} 0$ in $L^{2}(\Omega)^{n}$ which means that $\nabla_{y} u^{0}=0$, proving that actually $u^{0} \in H_{s}^{1}(\Omega)$.

On the other hand, since $\left\|f^{\epsilon}\right\|_{L^{2}(\Omega)} \leq c$, there exists $f^{0} \in L^{2}(\Omega)$ such that, up to a subsequence, $f^{\epsilon} \rightarrow f^{0}$ in $L^{2}(\Omega)$. Moreover, since

$$
\int_{\Omega}\left(\nabla_{x} u^{\epsilon} \cdot \nabla_{x} \varphi+u^{\epsilon} \varphi\right) d x d y \stackrel{\epsilon \rightarrow 0}{\longrightarrow} \int_{\Omega}\left(\nabla_{x} u^{0} \cdot \nabla_{x} \varphi+u^{0} \varphi\right) d x d y, \quad \forall \varphi \in H_{s}^{1}(\Omega),
$$

and

$$
\int_{\Omega}\left(\nabla_{x} u^{\epsilon} \cdot \nabla_{x} \varphi+u^{\epsilon} \varphi\right) d x d y=\int_{\Omega} f^{\epsilon} \varphi d x d y, \quad \forall \varphi \in H_{s}^{1}(\Omega),
$$

we obtain

$$
\int_{\Omega}\left(\nabla_{x} u^{0} \cdot \nabla_{x} \varphi+u^{0} \varphi\right) d x d y=\int_{\Omega} f^{0} \varphi d x d y, \quad \forall \varphi \in H_{s}^{1}(\Omega),
$$

that is, $u^{0}=A_{0}^{-1} f^{0}$.

In the following lemma, similar to [1, Lemma 3.1], we strengthen the previous convergence result.

Lemma 3.2. Let $f^{\epsilon}, u^{\epsilon}, f^{0}$ and $u^{0}$ be as in Lemma 3.1. Then

$$
\lim _{\epsilon \rightarrow 0}\left\|u^{\epsilon}-u^{0}\right\|_{H^{1}(\Omega)}=0 .
$$

Proof. It follows from the weak convergence, $u^{\epsilon} \rightarrow u^{0}$ in $H^{1}(\Omega)$ (obtained in Lemma 3.1), that

$$
\begin{aligned}
\left\|u^{0}\right\|_{H^{1}(\Omega)}^{2} & =\int_{\Omega}\left(\left|\nabla_{x} u^{0}\right|^{2}+\left|u^{0}\right|^{2}\right) d x d y \\
& \leq \lim _{\epsilon \rightarrow 0} \int_{\Omega}\left(\left|\nabla_{x} u^{\epsilon}\right|^{2}+\left|\nabla_{y} u^{\epsilon}\right|^{2}+\left|u^{\epsilon}\right|^{2}\right) d x d y \\
& \leq \lim _{\epsilon \rightarrow 0} \int_{\Omega}\left(\left|\nabla_{x} u^{\epsilon}\right|^{2}+\left|\nabla_{y} u^{\epsilon}\right|^{2}+\left|u^{\epsilon}\right|^{2}\right) d x d y \\
& \leq \lim _{\epsilon \rightarrow 0} \int_{\Omega}\left(\left|\nabla_{x} u^{\epsilon}\right|^{2}+\frac{1}{\epsilon^{2}}\left|\nabla_{y} u^{\epsilon}\right|^{2}+\left|u^{\epsilon}\right|^{2}\right) d x d y \\
& =\lim _{\epsilon \rightarrow 0} \int_{\Omega} f^{\epsilon} u^{\epsilon} d x d y \\
& =\int_{\Omega} f^{0} u^{0}=\int_{\Omega}\left(\left|\nabla_{x} u^{0}\right|^{2}+\left|u^{0}\right|^{2}\right) d x d y=\left\|u^{0}\right\|_{H^{1}(\Omega)}^{2},
\end{aligned}
$$

which proves the statement. 
Remark 3.3. Although we can obtain Theorem 2.1 immediately from Lemma 3.2, which is actually done in [1], we prefer to use the technique employed in [4-6] to obtain the expected convergence result. We think it is possible in this way to consider more classes of singular perturbations problems as reaction-diffusion equations in dumbbell type domains [4-6] and reaction-diffusion equations with large diffusion phenomena [8], besides estimating their rates of convergence [2].

Lemma 3.4. Let $f^{\epsilon}, u^{\epsilon}, f^{0}$ and $u^{0}$ be as in Lemma 3.1. If

$$
\begin{aligned}
& \lambda^{\epsilon}=\frac{1}{2}\left\|u^{\epsilon}\right\|_{\epsilon}^{2}-\int_{\Omega} f^{\epsilon} u^{\epsilon} d x d y=\min _{\varphi \in H^{1}(\Omega)}\left\{\frac{1}{2}\|\varphi\|_{\epsilon}^{2}-\int_{\Omega} f^{\epsilon} \varphi d x d y\right\}, \\
& \lambda^{0}=\frac{1}{2}\left\|u^{0}\right\|_{H^{1}(\Omega)}^{2}-\int_{\Omega} f^{0} u^{0} d x d y=\min _{\varphi \in H^{1}(\Omega)}\left\{\frac{1}{2}\|\varphi\|_{H^{1}(\Omega)}^{2}-\int_{\Omega} f^{0} \varphi d x d y\right\},
\end{aligned}
$$

then $\lim _{\epsilon \rightarrow 0} \lambda_{\epsilon}=\lambda_{0}$.

Proof. Taking $u^{0}$ as a test function for $\lambda^{\epsilon}$ and recalling that $\nabla_{y} u^{0}=0$,

$$
\begin{aligned}
\lambda^{\epsilon} & \leq \frac{1}{2}\left\|u^{0}\right\|_{H^{1}(\Omega)}^{2}-\int_{\Omega} f^{\epsilon} u^{0} d x d y \\
& =\frac{1}{2}\left\|u^{0}\right\|_{H^{1}(\Omega)}^{2}-\int_{\Omega} f^{0} u^{0} d x d y-\int_{\Omega}\left(f^{\epsilon}-f^{0}\right) u^{0} d x d y \\
& =\lambda^{0}-\int_{\Omega}\left(f^{\epsilon}-f^{0}\right) u^{0} d x d y,
\end{aligned}
$$

which leads to

$$
\limsup _{\epsilon \rightarrow 0} \lambda_{\epsilon} \leq \lambda_{0}
$$

On the other hand,

$$
\begin{aligned}
\lambda_{0}= & \frac{1}{2} \int_{\Omega}\left(\left|\nabla_{x} u^{0}\right|^{2}+\left|u^{0}\right|^{2}\right) d x d y-\int_{\Omega} f^{0} u^{0} d x d y \\
= & \frac{1}{2} \int_{\Omega}\left(\left|\nabla_{x} u^{0}-\nabla_{x} u^{\epsilon}+\nabla_{x} u^{\epsilon}\right|^{2}+\left|u^{0}-u^{\epsilon}+u^{\epsilon}\right|^{2}\right) d x d y-\int_{\Omega} f^{0}\left(u^{0}-u^{\epsilon}\right) d x d y \\
& \quad-\int_{\Omega}\left(f^{0}-f^{\epsilon}\right) u^{\epsilon} d x d y-\int_{\Omega} f^{\epsilon} u^{\epsilon} d x d y \\
= & \frac{1}{2} \int_{\Omega}\left(\left|\nabla_{x} u^{0}-\nabla_{x} u^{\epsilon}\right|^{2}+\left|u^{0}-u^{\epsilon}\right|^{2}\right) d x d y+\int_{\Omega}\left(\nabla_{x} u^{0}-\nabla_{x} u^{\epsilon}\right) \cdot \nabla_{x} u^{\epsilon} d x d y \\
& +\frac{1}{2} \int_{\Omega}\left|\nabla_{x} u^{\epsilon}\right|^{2} d x d y+\int_{\Omega}\left(u^{0}-u^{\epsilon}\right) u^{\epsilon} d x d y+\frac{1}{2} \int_{\Omega}\left|u^{\epsilon}\right|^{2} d x d y \\
& \quad-\int_{\Omega} f^{0}\left(u^{0}-u^{\epsilon}\right) d x d y-\int_{\Omega}\left(f^{0}-f^{\epsilon}\right) u^{\epsilon} d x d y-\int_{\Omega} f^{\epsilon} u^{\epsilon} d x d y \\
\leq & \frac{1}{2}\left\|u^{0}-u^{\epsilon}\right\|_{H^{1}(\Omega)}^{2}+\lambda_{\epsilon}+\int_{\Omega}\left(\nabla_{x} u^{0}-\nabla_{x} u^{\epsilon}\right) \cdot \nabla_{x} u^{\epsilon} d x d y+\int_{\Omega}\left(u^{0}-u^{\epsilon}\right) u^{\epsilon} d x d y \\
& \quad-\int_{\Omega} f^{0}\left(u^{0}-u^{\epsilon}\right) d x d y-\int_{\Omega}\left(f^{0}-f^{\epsilon}\right) u^{\epsilon} d x d y
\end{aligned}
$$




$$
\begin{gathered}
\leq \frac{1}{2}\left\|u^{0}-u^{\epsilon}\right\|_{H^{1}(\Omega)}^{2}+\lambda^{\epsilon}+\left\|\nabla_{x} u^{0}-\nabla_{x} u^{\epsilon}\right\|_{L^{2}(\Omega)}\left\|\nabla_{x} u^{\epsilon}\right\|_{L^{2}(\Omega)}+\left\|u^{0}-u^{\epsilon}\right\|_{L^{2}(\Omega)}\left\|u^{\epsilon}\right\|_{L^{2}(\Omega)} \\
+\left\|f^{0}\right\|_{L^{2}(\Omega)}\left\|u^{0}-u^{\epsilon}\right\|_{L^{2}(\Omega)}-\int_{\Omega}\left(f^{\epsilon}-f^{0}\right) u^{\epsilon} d x d y,
\end{gathered}
$$

which leads to $\lambda^{0} \leq \lim \inf _{\epsilon \rightarrow 0} \lambda^{\epsilon}$, proving the lemma.

Corollary 3.5 (Proof of Theorem 2.1). If $f^{\epsilon}, u^{\epsilon}, f^{0}$ and $u^{0}$ are as in Lemma 3.1 then

$$
\lim _{\epsilon \rightarrow 0}\left\|u^{\epsilon}-u^{0}\right\|_{\epsilon}=0
$$

Proof. Inspired by the proof of Lemma 3.4 and recalling that $\nabla_{y} u^{0}=0$,

$$
\begin{aligned}
\lambda^{\epsilon}= & \frac{1}{2}\left\|u^{\epsilon}\right\|_{\epsilon}^{2}-\int_{\Omega} f^{\epsilon} u^{\epsilon} d x d y \\
= & \frac{1}{2}\left\|u^{\epsilon}-u^{0}+u^{0}\right\|_{\epsilon}^{2}-\int_{\Omega} f^{\epsilon}\left(u^{\epsilon}-u^{0}\right) d x d y-\int_{\Omega}\left(f^{\epsilon}-f^{0}\right) u^{0} d x d y-\int_{\Omega} f^{0} u^{0} d x d y \\
= & \frac{1}{2}\left\|u^{\epsilon}-u^{0}\right\|_{\epsilon}^{2}+\frac{1}{2}\left\|u^{0}\right\|_{H^{1}(\Omega)}^{2}+\int_{\Omega}\left(\nabla_{x} u^{\epsilon}-\nabla_{x} u^{0}\right) \cdot \nabla_{x} u^{0} d x d y+\int_{\Omega}\left(u^{\epsilon}-u^{0}\right) u^{0} d x d y \\
& \quad-\int_{\Omega} f^{0} u^{0} d x d y-\int_{\Omega} f^{\epsilon}\left(u^{\epsilon}-u^{0}\right) d x d y-\int_{\Omega}\left(f^{\epsilon}-f^{0}\right) u^{0} d x d y \\
= & \frac{1}{2}\left\|u^{0}-u^{\epsilon}\right\|_{\epsilon}^{2}+\lambda^{0}+\int_{\Omega}\left(\nabla_{x} u^{0}-\nabla_{x} u^{\epsilon}\right) \cdot \nabla_{x} u^{\epsilon} d x d y+\int_{\Omega}\left(u^{0}-u^{\epsilon}\right) u^{\epsilon} d x d y \\
& \quad-\int_{\Omega} f^{\epsilon}\left(u^{\epsilon}-u^{0}\right) d x d y-\int_{\Omega}\left(f^{\epsilon}-f^{0}\right) u^{0} d x d y .
\end{aligned}
$$

The result follows by arguing as in Lemma 3.4, recalling that $\lambda^{\epsilon} \stackrel{\epsilon \rightarrow 0}{\longrightarrow} \lambda^{0}$.

\section{Acknowledgements}

The author thanks Professor Alexandre Carvalho and Professor Jose Arrieta for many stimulating conversations and useful remarks. The author also thanks the anonymous referee for careful reading and suggestions which led to an improvement of the text.

\section{References}

[1] F. Antoci and M. Prizzi, 'Reaction-diffusion equations on unbounded thin domains', Topol. Methods Nonlinear Anal. 18 (2001), 283-302.

[2] J. M. Arrieta, F. D. M. Bezerra and A. N. Carvalho, 'Rate of convergence of attractors for some singularly perturbed parabolic problems', Topol. Methods Nonlinear Anal. (2013), to appear.

[3] J. M. Arrieta and A. N. Carvalho, 'Spectral convergence and nonlinear dynamics of reactiondiffusion equations under perturbations of the domain', J. Differential Equations 199 (2004), 143-178.

[4] J. M. Arrieta, A. N. Carvalho and G. Lozada-Cruz, 'Dynamics in dumbbell domains I. Continuity of the set of equilibria', J. Differential Equations 231(2) (2006), 551-597. 
[5] J. M. Arrieta, A. N. Carvalho and G. Lozada-Cruz, 'Dynamics in dumbbell domains II. The limiting problem', J. Differential Equations 247 (2009), 174-202.

[6] J. M. Arrieta, A. N. Carvalho and G. Lozada-Cruz, 'Dynamics in dumbbell domains III. Continuity of Attractors', J. Differential Equations 231 (2009), 225-259.

[7] J. M. Arrieta, A. N. Carvalho, M. C. Pereira and R. P. Silva, 'Semilinear parabolic problems in thin domains with a highly oscillatory boundary', Nonlinear Anal. 74 (2011), 5111-5132.

[8] V. L. Carbone, A. N. Carvalho and K. Schiabel-Silva, 'Continuity of attractors for parabolic problems with localized large diffusion', Nonlinear Anal. 68 (2008), 515-535.

[9] A. N. Carvalho and S. Piskarev, 'A general approximation scheme for attractors of abstract parabolic problems', Numer. Funct. Anal. Optim. 27(7-8) (2006), 785-829.

[10] I. S. Ciuperca, 'Reaction-diffusion equations on thin domains with varying order of thinness', J. Differential Equations 126 (1996), 244-291.

[11] T. Elsken, 'Limiting behavior of attractors for systems on thin domains', Hiroshima Math. J. 32 (2002), 389-415.

[12] T. Elsken, 'Attractors for reaction-diffusion equations on thin domains whose linear part is nonselfadjoint', J. Differential Equations 206 (2004), 94-126.

[13] T. Elsken, 'A reaction-diffusion equation on a net-shaped thin domain', Studia Math. 165 (2004), 159-199.

[14] T. Elsken, 'Continuity of attractors for net-shaped thin domain', Topol. Methods Nonlinear Anal. 26 (2005), 315-354.

[15] J. K. Hale and G. Raugel, 'Reaction-diffusion equations on thin domains', J. Math. Pures Appl. 71(1) (1992), 33-95.

[16] M. Prizzi, M. Rinaldi and K. P. Rybakowski, 'Curved thin domains and parabolic equations', Studia Math. 151 (2002), 109-140.

[17] M. Prizzi and K. P. Rybakowski, 'The effect of domain squeezing upon the dynamics of reaction-diffusion equations', J. Differential Equations 173 (2001), 271-320.

[18] G. Raugel, Dynamics of Partial Differential Equations on Thin Domains, Lecture Notes in Mathematics, 1609 (Springer, Berlin, Heidelberg, 1995).

[19] A. M. Rekalo, 'Asymptotic behavior of solutions of nonlinear parabolic equations on two-layer thin domains', Nonlinear Anal. 52 (2003), 1393-1410.

\section{RICARDO P. SILVA, Instituto de Geociências e Ciências Exatas, UNESP - Univ Estadual Paulista, Departamento de Matemática, 13506-900, Rio Claro SP, Brazil e-mail: rpsilva@rc.unesp.br}

